\title{
Influencia de la Atención Sostenida en la Lectura Comprensiva en los Niños y Niñas de Ocho Años de Edad del Centro de Educación General Básica "La Providencia” en la Ciudad de Ambato.
}

Influence of Sustained Attention in Reading Comprehension in Children Eight Years Old Center General Basic Education "Providence" in the city of Ambato

\author{
Verónica Fernanda Flores Hernández ${ }^{1}$ \\ Rubén Israel Yánez Sánchez ${ }^{2}$ \\ ${ }^{1}$ Universidad Central del Ecuador (UCE) \\ Bolivia s/n y Eustorgio Salgado \\ Quito-Ecuador \\ Email: verifer123@hotmail.com
}

\begin{abstract}
Resumen
Tesis sobre Neuropsicología Infantil, especificamente, influencia de la atención sostenida en la lectura comprensiva en niños de 8 años. El objetivo fundamental de la presente investigación es comprobar que tanta influencia tienes la atención sostenida sobre la lectura comprensiva, sabiendo que los dos procesos en forma individual son esenciales en el aprendizaje. El problema identificado en los niños y niñas de ocho años de edad, pertenecientes a la institución donde se realizó el estudio es la falta de comprensión en los textos que leen, lo que genera un bajo rendimiento escolar. La hipótesis menciona: La madurez de la atención sostenida influye en el desarrollo de la lectura comprensiva en niños y niñas de ocho años de edad que asisten al centro de educación general básica La Providencia de la cuidad de Ambato, en el año lectivo 2012 - 2013". Dentro de la metodología se ha utilizado la aplicación de ENI. La conclusión se ha detallado en base a los resultados obtenidos que muestran que la madurez de la atención sostenida influye en el desarrollo de la lectura comprensiva.
\end{abstract}

Palabras Claves: Atención; atención sostenida lectura; comprensión, rendimiento.

\begin{abstract}
Thesis on Child Neuropsychology, specifically, influence of sustained attention in reading comprehension in 8 year old children. The fundamental aim of this present research is to verify how much influence the sustained attention has on reading comprehension, knowing that both processes individually are essential in learning. The problem identified in boys and girls of eight years of age, belonging to the institution where the study was conducted is the lack of understanding of the texts they read, which generates a low school performance. The hypothesis states: The maturity of sustained attention influences the development of reading comprehension in boys and girls of eight years of age who attend the center of basic general education The Providence of Ambato city, in the academic year 2012 - 2013 ". Within the methodology the application of ENI has been used. The conclusion has been detailed on the basis of the obtained results that show that the maturity of sustained attention influences the development of reading comprehension.
\end{abstract}

Keywords: Attention; Reading sustained attention; comprehension performance. 


\section{Introducción}

Los problemas de aprendizaje forman parte de una temática poco estudiada en nuestro medio, aunque el Ministerio de Educación Pública ha abordado este tema desde las destrezas del hablar, escuchar leer y escribir, mismas que pueden ser desarrolladas por los infantes.

En el ámbito escolar se observa con mayor frecuencia dificultades en ortografía, lectura, matemáticas, idiomas presumiendo que podría encontrarse además afectada la atención, concentración y memoria.

Battro en el año 2005, menciona la importancia de la atención en los diferentes procesos educativos que atraviesan los infantes.

Según Ardila y Ostrosky que plantean un modelo cognoscitivo cuyos correlatos neuroanatómicos están asociados a los dominios cognoscitivos de la atención, se propone que la atención es un proceso que aún no tiene una definición estándar y universalmente aceptada.

El proceso atencional selectivo que ocurre en respuesta a la capacidad de procesamiento limitado es aquel que se busca enlazar en el proceso lector de los niños de 8 años. (Heilman, 2002). Según la Academia de Ciencias Luventicus (@2002-2011) se entiende que la lectura comprensiva es indispensable para el estudiante. Esto es algo que él mismo va descubriendo a medida que avanza en sus estudios. En el nivel primario a veces alcanza con una comprensión mínima y una buena memoria altas calificaciones, sobre todo si a ello se suman prolijidad, atención y buena conducta. La misma academia menciona que al pensar relacionamos conceptos, datos e informaciones, estableciendo entre ellos relaciones causales o comparaciones, clasificándolos, reuniéndolos bajo una explicación general que los engloba y los supera. Leer comprensivamente es leer entendiendo a qué se refiere el autor con cada una de sus afirmaciones y cuáles son los nexos, las relaciones que unen dichas afirmaciones entre sí. Es por ello que al enfocar atención en detalles del texto se puede comprender el mensaje del mismo. Según Vargas Fuentes Limena (especialista en trastornos del lenguaje- 2011) la atención es la capacidad mental para fijarse en uno o varios aspectos de la realidad y prescindir de los restantes, implica un acto que requiere la energía para poder delimitar ciertas actividades de carácter sensorial, tendientes a producir una mejor visión de un objeto o una situación. Por ello, se ha encaminado la presente investigación al estudio de la atención sostenida y su influencia en la lectura comprensiva en niños y niñas de 8 años de edad del Centro de Educación General Básica "La Providencia”.

\section{Materiales y Métodos}

Se trata de un estudio basado en dos reactivos el ENI y Neuropsi, mismos que han sido utilizados para evaluar lectura comprensiva y atención sostenida respectivamente.

De inicio se solicitó la autorización respectiva de cada uno de los padres de familia y de las respectivas autoridades de la institución donde se realizó la investigación.

Posterior a ello se solicitó información de la profesora encargada de dichos niños en estudio, la misma que supo manifestar que una e las dificultades más significativas en los infantes de ocho años de edad en el proceso de lectura comprensiva, en la respectiva entrevista se pudo notar que los profesionales de la educación no tienen claro las causas de este posible fenómeno particular por común en los estudiantes por lo cual se procede a realizar la evaluación en base a los test antes mencionados.

Posterior a lo mencionado se procede a la aplicación del Test ENI literal correspondiente a la lectura comprensiva y NEUROPSI, literal correspondiente a la atención sostenida, cada uno de ellos fueron aplicados en forma individual utilizando alrededor de 17 minutos para cada estudiante.

Posterior a la evaluación se procede a obtener los percentiles de cada uno de los niños, con estos resultados se tabular la información.

Para el análisis de los datos obtenidos el procedimiento que se utilizó permitirá concordar los resultados obtenidos de las poblaciones encuestadas, con los resultados teóricos esperados, para medir el nivel de correlación que existe entre las frecuencias observadas y esperadas, se utilizó el estadístico Chi cuadrado $\mathrm{x}^{2}$.

Este método emplea los datos obtenidos al investigar, que a un nivel de significación se está dispuesto a aceptar la hipótesis alterna y si la opinión de decisión es menor se considere a la hipótesis nula.

Para la prueba de la hipótesis en la que se cuenta con frecuencias tanto absolutas como relativas en las tablas estadísticas de las encuestas, se procede a realizar la prueba del chi- cuadrado $\left(\mathrm{x}^{2}\right)$, que permitirá establecer el conjunto de frecuencias esperadas teóricas si se aplica la fórmula.

$\mathrm{X}^{2}=\sum \frac{(O-E)^{2}}{E}$

\section{Resultados}

Con el fin de comprobar la hipótesis "La madurez de la atención sostenida influye en el desarrollo de la lectura comprensiva en niños y niñas de ocho años de edad que asisten al centro de educación general básica La Providencia de la ciudad de Ambato, en el año lectivo 2012 - 2013”, se procede a tabular los resultados, donde se obtiene los siguientes datos:

- La aplicación de herramientas determinó que tanto niñas como niños si tienen lectura 
comprensiva en su gran porcentaje presentan percentiles mayores a 70. (Cuadro1)

- $\quad$ La puntuación es normal en niños y niñas en la prueba de atención sostenida (Dígitos). (Cuadro 2)

- En la prueba de series sucesivas, correspondiente a atención sostenida se evidencia un $25 \%$ de niñas evaluadas como normal alto, un $75 \%$ evaluadas como normal. En niños un 38\% como normal alto y un 62\% como normal. (Cuadro 3)

Tabla 1

NIÑOS

LECTURA COMPRENSIVA

\begin{tabular}{|c|c|c|}
\hline RESULTADOS DERCENTILES & NUMERO DE CASOS & DOACENIAEE \\
\hline MeNos $x$ & 0 & os \\
\hline ENTRE 70.80 & 12 & \\
\hline ENTRE 81.90 & 12 & 306 \\
\hline ENTRE 91.100 & 16 & 405 \\
\hline $\begin{array}{ll}\text { TOTAL } \\
\text { TOL }\end{array}$ & $\frac{16}{40}$ & $\frac{4005}{1005}$ \\
\hline
\end{tabular}

NIÑAS

\section{LECTURA COMPRENSIVA}

\begin{tabular}{|c|c|c|}
\hline RESULTADOS PERCENTILES & NUMERO DECASOS & POACENTANE \\
\hline MENOS 70 & 1 & $3 \%$ \\
\hline ENTRE 70.80 & 14 & $35 \%$ \\
\hline ENTRE 81.90 & 7 & $18 \%$ \\
\hline ENTRE 91.100 & 18 & $45 \%$ \\
\hline TOTAL & 40 & $100 \%$ \\
\hline ATENCIÓN SOSTENIDA & \multicolumn{2}{|l|}{ NIÑos } \\
\hline \multicolumn{3}{|l|}{ PRUEBA DE DIGITOS } \\
\hline OPCIONES EVALUACION & FRECUENCIAS & \begin{tabular}{|l} 
PORCENTAUE \\
\end{tabular} \\
\hline PUNTUACCÓN NORMALZZAOA DE 7 A II ES NORMAL & 40 & $100 \%$ \\
\hline 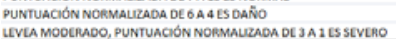 & $\vdots$ & $\begin{array}{l}0 \% \\
0 \%\end{array}$ \\
\hline TOTAL & 40 & 1000 \\
\hline
\end{tabular}
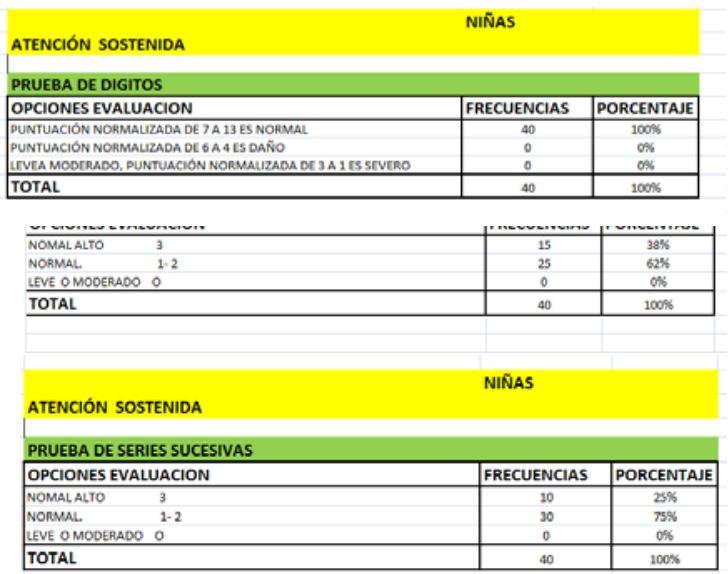

Tomando en cuenta los datos mencionados se aplica la fórmula anteriormente mencionada obteniendo como resultado final:

Figura 1

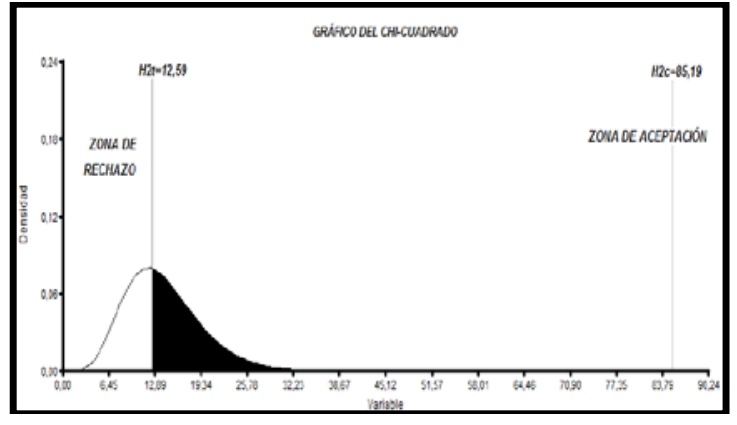

La aceptación de la hipótesis alterna, es decir, La madurez de la atención sostenida influye en el desarrollo de la lectura comprensiva en niños y niñas de ocho años de edad que asisten al centro de educación general básica La Providencia de la cuidad de Ambato, en el año lectivo 2012 - 2013”

\section{Conclusiones}

La investigación realizada se ha basado en la necesidad de brindar apoyo a la diferentes falencias encontradas al nivel de comprensión lectora en los niños y niñas de ocho años de edad que asisten al Centro de Educación General Básica "La Providencia” en la ciudad de Ambato.

Para el respectivo estudio se ha tomado como referencia teórica a las Unidades Cerebrales de Luria, mismo que menciona tres unidades cerebrales:

\section{- $\quad$ Primera Unidad Cerebral}

La primera unidad funcional, según Luria, es un aparato que mantiene el tono cortical y el estado de vigilia y que regula estos estados de acuerdo con las demandas que en ese momento confronta el organismo, por lo tanto, trabaja en estrecha cooperación con los niveles superiores del córtex, el estado de vigilia es el primer requisito para que ocurra cualquier actividad organizada, ya que sólo bajo condiciones óptimas de vigilia es posible que el hombre reciba y analice la información, ya que el tono cortical desaparece durante el sueño, pues solamente en ella los procesos mentales siguen un curso corrector, para el cual es necesario tener un nivel optimó de tono cortical. Luria destaca tres fuentes primarias de activación:

a) Primera: Procesos metabólicos del organismo, "economía interna”. Estos procesos en sus formas más simples, están conectados con los procesos respiratorios y digestivos; regulados principalmente por el hipotálamo. Otras formas más complejas de este tipo de activación están relacionadas con los procesos metabólicos organizados de ciertos sistemas conductuales innatos; conducta instintiva, sexual y de alimentación. 
b) Segunda: Reflejo de orientación, esta conectada con la llegada de estímulos del mundo exterior del cuerpo y conduce a la producción de formas completamente diferentes de activación.

Este es el único tipo de mecanismo que puede permitir procesos de habituación, de forma que unos estímulos repetidamente presentados pierden su novedad, y la movilización especial del organismo cuando aparecen no es necesaria mente de larga duración.

c) Tercera: Lenguaje, una gran parte de la actividad humana se evoca por intenciones y planes (metas), que se forman durante la vida conciente del hombre y que son esenciales en la motivación y que efectúan con la íntima participación, inicialmente externa, y mas adelante interna, del lenguaje.

Como conclusión se puede mencionar que la primera unidad funcional constituye parte importante en los distintos procesos mentales, ya que mantiene un estado de arousal apropiado que permite centrar la atención sostenida que se la define como la forma más compleja atención

- Segunda Unidad Cerebral La segunda unidad funcional comprende regiones corticales específicas en los hemisferios izquierdo y derecho, incluido el cuerpo calloso (Lóbulos parietal, temporal y occipital). La recepción sensorial y el análisis de datos se realizan en ambos hemisferios con objetivos diferentes. La organización espacial (dibujos y gestos) son función del hemisferio derecho, mientras que la simbolización y el lenguaje son del hemisferio izquierdo

- $\quad$ Tercera Unidad Cerebral. La tercera unidad cerebral corresponde al funcionamiento cognitivo de orden superior (Planificación y Ejecución). Los lóbulos frontales y la corteza motora son los órganos implicados en la programación. El área prefrontal del cerebro tiene conexiones con los demás sistemas y ejerce un control ejecutivo (programación, regulación y verificación de la información). (1)

Para el estudio de la Lectura se ha tomado como guía el Modelo Neuropsicológico Funcional de la Lectura, que proporciona una visión analítica del contenido del texto, este tipo de lectura tiene por objeto la interpretación y comprensión crítica del texto por lo cual es capaz de decodificar el mensaje mediante interrogación, análisis o crítica.

Dicho modelo además nos 4 puntos de partida:

1. El input visual llega a las áreas primarias en la corteza visual del lóbulo occipital como cualquier información visual (en este caso gráficos - letras)

2. El sistema de análisis visual se encarga de identificar y codificar las letras. "El reconocimiento global se utilizaría para las palabras conocidas, mientras que el análisis de letra a letra para las palabras desconocidas." (Puyuelo y Rondal, 2003)

3. La información del sistema de análisis visual puede seguir dos caminos distintos. Si llega al almacén de reconocimiento visual se compara con las palabras existentes en él y se identifican las palabras. Aquí se inicia la llamada ruta léxica de la lectura. En cambio, si la información del análisis visual pasa directamente a la trans-codificación grafema-fonema se inicia la llamada ruta fonológica.

- Ruta Léxica. Parte del almacén visual de las palabras conocidas; por lo que permitirá solo leer las palabras que pertenecen a ese vocabulario visual. Las palabras que son reconocidas pasan al sistema semántico donde se les otorga el significado. Las palabras comprendidas y cargadas de su significado se pueden leer en voz alta siempre y cuando estén disponibles en el almacén de producción del léxico oral y desde aquí se depositará en el sistema fonémico o "almacén de pronunciación" preparadas para ser emitidas. Pero, puede darse el caso de que directamente del almacén de reconocimiento visual, una vez identificada la palabra, se dirija al almacén de producción del léxico oral sin acceder al sistema semántico produciéndose la lectura de palabras sin comprensión.

- Ruta Fonológica. Mediante esta ruta se explica la lectura de las palabras no familiares y la lectura de pseudopalabras. Una vez identificadas las letras que componen la palabra en el sistema de análisis visual se recupera el sonido que corresponde a cada una de esas letras por medio del mecanismo llamado transcodificación grafema-fonema. Esta representación de la producción de la palabra se compara con el léxico de reconocimiento oral y se procederá a continuación como si han se tratara de una palabra oral relacionándose de esta manera con el sistema semántico para llegar a acceder al significado. También se pueden leer palabras no conocidas y pseudopalabras por lo que directamente de la conversión grafema-fonema se accede al sistema fonémico y se procede a la articulación de la palabra. Cuando se está leyendo en voz alta y también durante el habla se realizan correcciones, lo que supone una conexión directa entre el output (palabra oral) y el input (palabra oída). El hablante o lector recibe constantemente el feedback de sus palabras. La existencia de este proceso de feedback es muy evidente en la lectura en voz alta de niños con dificultades lectoras que realizan numerosas rectificaciones mientras leen, puesto que al comparar su palabra incorrecta con el almacén léxico se rechaza inmediatamente para sustituirse por la palabra correcta y continuar de esta forma los demás pasos del proceso.

En base a todo lo mencionado se concluye que la comprensión de los textos supone mucho más que la comprensión aislada de palabras, así que el sistema léxico se relaciona directamente con el 
sistema fonológico, la comprensión global y significativa del texto supone la puesta en funcionamiento de procesos cognitivos superiores que implican la identificación de las ideas principales del texto, su relación con los conocimientos previos y la posibilidad de realizar inferencias, todo ello expresado en términos generales en el sistema ejecutivo.

En base a estas dos teorías que han regido la investigación se aplicó a toda la población el test ENI para evaluar lectura comprensiva y NEUROPSI para evaluar atención, ante lo cual, los resultados muestran que los niveles de madurez atencional afectan significativamente al proceso de lectura comprensiva de dichos infantes.

Además se observa la afectación con mayor prevalencia en niños que en niñas, lo cual nos puede dar un indicio que el proceso de madurez atencional en los infantes varones podría ser más lento que en las infantes mujeres.

\section{Agradecimientos}

A la Dra. María Elena Silva por su valioso aporte y guía en la investigación y a todos que de una u otra forma colaboraron para realizar el presente trabajo.

A la Dra. Rocío Ponce por su colaboración y ayuda en la elaboración del presente artículo.

\section{Referencias}

[1] Ardila, A. Roselli, M. Matute, E. 2005.

Neuropsicología de los Trastornos de Aprendizaje. México DF. Editorial El Manual Moderno

[2] Portellano, J. A 2008. Neuropsicología Infantil. Madrid- España. Editorial Síntesis.

[3] Gil, R. 1999. Manual De Neuropsicología. España: Editorial MASSON S.A

[4] Balarezo L, Mancheno S. 1996. Compendio De Neuropsicología.

[5]Ostrosky F. 2007. ¿Problemas de lenguaje?

México: Editorial LP

[6]Luria, A. R. 1979.

El cerebro en acción. Barcelona: Fontanella (1)

[7] Psicopedagogía.com 